\title{
SINGLE BUNCH LONGITUDINAL INSTABILITIES IN PROTON STORAGE RINGS
}

\author{
J. Gao, LAL, B.P. 34, F-91898 Orsay cedex, France
}

\section{Abstract}

In this paper we try to explain the single bunch energy spread increasing in a proton storage ring. Different from an electron machine, the random motions of some particles of a bunch are caused by the nonlinear synchrotron oscillations perturbed by the short range longitudinal wakefield. An analytical single bunch threshold current formula has been established to indicate the beginning of the longitudinal random motions. By using kinetic descriptions one shows that the energy spread of the particles which execute random motions will increase, and the energy spectrum width of the random motions will be enlarged with increasing bunch current.

\section{INTRODUCTION}

The problem of the longitudinal instabilities in a proton storage ring is quite different from that in an electron one where the synchrotron radiation plays an important role [1]. It is observed experimentally that below an apparent threshold bunch current the bunch length increases with increasing current while the bunch energy spread remain unchanged, and the mechanism of this bunch lengthening is explained quite well by the potential well distortion theory. When the bunch current surpasses the threshold value the energy spread of the bunch increases also. In this paper, we try to explain the mechanism of the single bunch energy spread increasing in a proton storage ring and establish an analytical formula for the threshold current.

\section{LONGITUDINAL MOTION PERTURBED BY SHORT RANGE WAKEFIELD}

In a circular proton storage ring a non-synchronous particle will oscillate around the the synchronous one under the rf focusing in the longitudinal direction, and its phase $\phi$ with respect to the rf accelerating potential is determined by the following differential equation [2]:

$$
\frac{d^{2} \phi}{d t^{2}}+\frac{\Omega_{s}^{2}}{\cos \phi_{s}}\left(\sin \phi-\sin \phi_{s}\right)=0
$$

and

$$
\Omega_{s}^{2}=\frac{e \hat{V} h \eta \omega_{s} \cos \phi_{s}}{2 \pi R_{s} p_{s}}
$$

where $\hat{V}$ is the peak accelerating voltage, $h$ is the harmonic number, $\eta=1 / \gamma^{2}-\alpha, \gamma$ is the normalized particle energy, $\alpha$ is the momentum, $R_{s}$ is the average radius of the ring, $\omega_{s}=c / R_{s}, p_{s}$ and $\phi_{s}$ are the momentum and the phase of the synchronous particle. For the convenience in the later mathematical treatment, we approximate eq. 1 by the following differential equation

$$
\frac{d^{2} \Delta \phi}{d t^{2}}+\Omega_{s}^{2} \sin \Delta \phi=0
$$

where $\Delta \phi=\phi-\phi_{s}$. For the stationary bucket eq. 1 and eq. 3 are equivalent. It is obvious that $\Delta \phi$ oscillates as a pendulum. The deviation of a particle with respect to the synchronous one in terms of energy is expressed as follows:

$$
\Delta E=-\frac{R_{s} p_{s}}{h \eta} \frac{d \Delta \phi}{d t}
$$

By defining $P=\frac{d \Delta \phi}{d t}$ and $Q=\Delta \phi$, eq. 3 can be derived from an Hamiltonian $H(Q, P, t)$ expressed as

$$
H(Q, P, t)=\frac{1}{2} P^{2}-\Omega^{2} \cos Q
$$

where $P$ and $Q$ are canonical. Let us change $P$ and $Q$ to the action-angle variables, $I$ and $\theta$. By introducing two variables $(\tilde{N}$ and $\xi)$

$$
\tilde{N}^{2}=\frac{1}{2}\left(1+\frac{H}{\Omega_{s}^{2}}\right)
$$

and

$$
\tilde{N} \sin \xi=\sin \frac{Q}{2},(\tilde{N} \leq 1)
$$

one gets [3]

$$
\begin{gathered}
I(H)=\frac{8}{\pi} \Omega_{s}\left(E\left(\frac{\pi}{2} ; \tilde{N}\right)-\left(1-\tilde{N}^{2}\right) F\left(\frac{\pi}{2} ; \tilde{N}\right)\right),(\tilde{N} \leq 1) \\
\theta=\frac{\partial S(Q, I)}{\partial I}
\end{gathered}
$$

with

$S(Q, I)=4 \Omega_{s}\left(E(\xi ; \tilde{N})-\left(1-\tilde{N}^{2}\right) F(\xi ; \tilde{N})\right),(\tilde{N} \leq 1)$

where $F(\xi ; \tilde{N})$ and $E(\xi ; \tilde{N})$ are the first and second kind elliptical integrals, respectively. The frequency of this nonlinear oscillator can be obtained easily as

$$
\Omega(H)=\frac{d H(I)}{d I}=\frac{\pi \Omega_{s}}{2 F\left(\frac{\pi}{2} ; \tilde{N}\right)},(\tilde{N} \leq 1)
$$

It is obvious that on the separatrix $(\tilde{N}=1)$ one has $H=$ $H_{c}=\Omega_{s}^{2}$, and $\Omega\left(H_{c}\right)=0$. Due to the interaction between the charged particles and the environment, after each turn a bunch will loss energy $W=e^{2} N_{p}^{2} \mathcal{K}_{/ /}^{\text {tot }}\left(\sigma_{z}\right)$, where $N_{p}$ is the number of particles in the bunch, $\mathcal{K}_{/ /}^{\text {tot }}\left(\sigma_{z}\right)$ is the total longitudinal loss factor of one turn, and $\sigma_{z}$ is the bunch 
length (here we assume that particles are relativistic, otherwise, $\mathcal{K}_{/ /}^{\text {tot }}\left(\sigma_{z}\right)$ will depend on the particle velocity, and space charge forces should be taken into account). This energy loss will be compensated by the rf cavities. Since the short range longitudinal wakefield varies within the bunch and the synchrotron oscillation period is much longer than that of one revolution, the additional energy variation of a particle due to the short range wakefield after each turn can be reasonably expressed as

$$
d E=\mathcal{U}_{w} \cos \theta=e^{2} N_{p} \mathcal{K}_{/ /}^{t o t}\left(\sigma_{z}\right) \cos \theta
$$

Obviously, averaging over one synchrotron period one has $<d E>=0$. Taking into account this additional energy variation after each revolution and the fact that this happens at instants $t_{k}$ with constant interval $T_{0}$ ( $T_{0}$ is the revolution period), a new Hamiltonian can be represented in the form:

$$
\begin{gathered}
H(I, \theta, t)^{*}=H(I)+\frac{1}{2} \Delta P^{2} T_{0} \sum_{k=-\infty}^{\infty} \delta\left(t-k T_{0}\right) \\
=H(I)+\frac{(d E)^{2} h^{2} \eta^{2}}{2 R_{s}^{2} p_{s}^{2}} T_{0} \sum_{k=-\infty}^{\infty} \delta\left(t-k T_{0}\right) \\
=H(I)+\frac{\mathcal{U}_{w}^{2} h^{2} \eta^{2} \cos ^{2} \theta}{2 R_{s}^{2} p_{s}^{2}} T_{0} \sum_{k=-\infty}^{\infty} \delta\left(t-k T_{0}\right)
\end{gathered}
$$

where we have omitted the crossing term coming from eq. 4 and eq. 12 since both quantities are statistically independent. Eq. 13 can be simplified as

$$
\begin{aligned}
& H(I, \theta, t)^{*}=H(I)+\Delta H T_{0} \sum_{k=-\infty}^{\infty} \delta\left(t-k T_{0}\right) \\
= & H(I)+\frac{\mathcal{U}_{w}^{2} h^{2} \eta^{2} \cos 2 \theta}{4 R_{s}^{2} p_{s}^{2}} T_{0} \sum_{k=-\infty}^{\infty} \delta\left(t-k T_{0}\right)
\end{aligned}
$$

where a constant term has been dropped, and

$$
\Delta H=\Delta H_{0} \cos 2 \theta=\frac{\mathcal{U}_{w}^{2} h^{2} \eta^{2}}{4 R_{s}^{2} p_{s}^{2}} \cos 2 \theta
$$

Consequently, one has

$$
\begin{gathered}
\frac{d I}{d t}=-\frac{\partial \Delta H}{\partial \theta} T_{0} \sum_{k=-\infty}^{\infty} \delta\left(t-k T_{0}\right) \\
\frac{d \theta}{d t}=\Omega(I)+\frac{\partial \Delta H}{\partial I} T_{0} \sum_{k=-\infty}^{\infty} \delta\left(t-k T_{0}\right)
\end{gathered}
$$

Since the kicks on the pendulum $H(I)$ repeat after constant time interval $T_{0}$, and between the kicks the motion is known, one can replace eqs. 16 and 17 by a so-called universal mapping:

$$
\begin{gathered}
I_{n+1}=I_{n}-T_{0} \frac{\partial \Delta H}{\partial \theta} \\
\theta_{n+1}=\theta_{n}+\Omega_{s} T_{0}+\Omega^{\prime} I_{n+1} T_{0}
\end{gathered}
$$

where $\Omega^{\prime}=d \Omega / d I$. We transform then the universal mapping into standard mapping which is expressed as

$$
\begin{gathered}
J_{n+1}=J_{n}+K_{0} \sin \Psi \\
\Psi_{n+1}=\Psi_{n}+J_{n+1}
\end{gathered}
$$

where $\Psi=2 \theta, J=2 T_{0} \Omega^{\prime} I, K_{0}=4 \Omega^{\prime} T_{0}^{2} \Delta H_{0}$, and a constant term has been omitted from eq. 21. At this stage we can discuss the condition on which there starts to have chaotic motions. To this end we can use the Chirikov criterion [4] which shows that when

$$
\left|K_{0}\right| \geq 1(0.97)
$$

the Kolmogorov-Arnold-Moser (KAM) invariant tori will be broken and the particles which satisfy this condition will move in a random way. From eq. 22 one gets the threshold bunch current of the onset of the stochastic motion

$$
I_{b, t h}=\frac{R_{s} p_{s}}{e \sqrt{\left|\Omega^{\prime}\right|} T_{0}^{2} h|\eta| \mathcal{K}_{/ /}^{t o t}\left(\sigma_{z}\right)}
$$

Taking advantage of our simplifying the longitudinal motion of a particle to a pendulum, we can have the analytical expression for $\Omega^{\prime}$. When a particle moves near the separatrix, one gets

$$
\left|\Omega^{\prime}\right|=\frac{1}{\pi^{4}\left|1-H_{b} / H_{c}\right|}\left(\ln \frac{32}{\left|1-H_{b} / H_{c}\right|}\right)^{3}
$$

where

$$
\begin{gathered}
\frac{H_{b}}{H_{c}}=\left(\frac{\delta E_{b}}{\delta E_{\text {max }}}\right)^{2}=\frac{\pi h|\eta| E_{s}}{\beta^{2} \hat{e V G}\left(\phi_{s}\right)}\left(\delta E_{b}\right)^{2} \\
G\left(\phi_{s}\right)=2 \cos \phi_{s}-\left(\pi-2 \phi_{s}\right) \sin \phi_{s}
\end{gathered}
$$

$H_{b}$ and $\delta E_{b}$ are the maximum Hamiltonian value and the relative energy spread of the bunch, $\delta E_{\max }$ is the maximum acceptance of the $\mathrm{rf}$ bucket in terms of relative energy spread, $\beta$ and $E_{s}$ are the normalized velocity and the energy of the particle, respectively.

\section{ENERGY SPREAD INCREASING}

For those particles which have already executed chaotic motions a kinetic description of them will be appropriate. We will loss, certainly, some detailed information on the particle trajectories, this method, however, will help us to get useful physical results. When the random motion occurs eqs. 20 and 21 can be regarded as a Markov process, and in consequence, the possibility distribution function $F(t, I)$ is described by the Fokker-Planck equation:

$$
\frac{\partial F}{\partial t}=-\frac{\partial(\mathcal{A} F)}{\partial I}+\frac{1}{2} \frac{\partial^{2}(\mathcal{D} F)}{\partial I^{2}}
$$

where $\mathcal{A}$ and $\mathcal{D}$ are defined as follows:

$$
\mathcal{A}=\frac{1}{2 \pi T} \int_{0}^{2 \pi} \Delta I d \theta
$$




$$
\mathcal{D}=\frac{1}{2 \pi T} \int_{0}^{2 \pi} \Delta I^{2} d \theta
$$

where $T$ is a small time interval. The legitimation of making average over the phase $\theta$ is based on the fact that the particle which moves randomly will mix its phase in the phase space. For our specific case described by eqs. 18 and 19 with $T=$ $T_{0}$, one has

$$
\begin{gathered}
\mathcal{A}=0 \\
\mathcal{D}=\frac{e^{2} N_{p}^{2} \mathcal{K}_{/ /}^{\text {tot }}\left(\sigma_{z}\right)^{2} h^{2} \eta^{2}}{2 R_{s}^{2} p_{s}^{2}} T_{0}
\end{gathered}
$$

Putting $\mathcal{A}=0$ and $\mathcal{D}$ into eq. 27 , one gets

$$
\frac{\partial F}{\partial t}=\frac{1}{2} \mathcal{D} \frac{\partial^{2} F}{\partial I^{2}}
$$

From eq. 32, one finds that stochastic heating occurs in the following way:

$$
<I^{2}>=I_{0}^{2}+\mathcal{D} t
$$

where $\left\langle I^{2}\right\rangle=\int_{0}^{\infty} I^{2} F d I$. Similarly, one finds

$$
\begin{aligned}
<\Delta E^{2}> & =\Delta E_{0}^{2}+\mathcal{D}_{e} t \\
\mathcal{D}_{e} & =\frac{\mathcal{U}_{w}^{2}}{2 T_{0}}
\end{aligned}
$$

Obviously, the amplitude of the energy deviations of those particles executing random motions will increase with time. From eq. 35 it is evident that the resistive part of the impedance of the machine determines the diffusion coefficient instead of the reactive part. The relevant effect of the interaction between the charged particles with the reactive part impedance is the potential well distortion which results in the reduction of the area of the rf bucket.

The distinction between an electron and a proton storage rings is that there exists always a stable (cold) core in the bunch of proton particles around the synchrotron particle where no energy spread increasing occurs.

\section{THE POWER SPECTRUM OF THE RANDOM MOTIONS}

As shown in section 2, when the bunch current surpasses the threshold current $I_{b, t h}$, some particles in the bunch will execute random motions, and the longitudinal positions of these particles will be random variables. When $I_{b} \gg I_{b, t h}$, the autocorrelator of these random motion has the form [3]

$$
\begin{gathered}
\mathcal{R}(t)=\mathcal{R}_{0} \exp \left(-\frac{t}{\tau_{c}}\right) \\
\tau_{c}=\frac{2 T_{0}}{\ln K_{0}} \\
=\frac{T_{0}}{\ln \left(\frac{\sqrt{\Omega^{\prime}} T_{0} e^{2} N_{p} \mathcal{K}_{/ 1}^{\text {tot }}\left(\sigma_{z}\right) h \eta}{R_{s} p_{s}}\right)}
\end{gathered}
$$

By virtue of Wiener-Khintchine theorem, we know that the spectral power density of the random variable is the Fourier transform of its autocorrelation function, and we have then

$$
\begin{aligned}
S(\omega)= & \frac{1}{2 \pi} \int_{-\infty}^{\infty} \exp (i \omega t) \mathcal{R}(t) d t \\
& =\frac{1}{\pi} \mathcal{R}_{0} \frac{\tau_{c}}{1+\omega^{2} \tau_{c}^{2}}
\end{aligned}
$$

The power spectrum $S(\omega)$ falls off rapidly when

$$
\omega>\omega_{c}=\frac{1}{\tau_{c}}
$$

The variation of $\omega_{c}$ with respect to the bunch current can be measured experimentally, and apparently, one has $\omega_{c} \propto$ $\ln \left(I_{b}\right)$.

\section{DISCUSSION ON THE BUNCH LENGTHENING}

In a proton machine, a bunch suffers from potential well distortion induced bunch lengthening just like what happens in an electron storage ring. When $I_{b} \geq I_{b, t h}$ some particles in the bunch will execute random motions and the synchrotron oscillation amplitudes of these particles will increase (but not those of the particles in the stable core). In a global point of view, the bunch length increases much more quickly with respect to the increasing bunch current compared with when $I_{b}<I_{b, t h}$. Since not all particles participate the random motions, it is much more difficult to get some simple formulae or equations to describe the global bunch lengthening and the energy spread increasing for the whole bunch current range as what has been done in ref. 1 for the electron storage rings.

\section{CONCLUSION}

In a proton storage ring, it is shown that the nonlinear longitudinal motion perturbed by the short range longitudinal wakefield can change the regular synchrotron motions of some particles in a bunch into random ones. An analytical formula of the threshold current for the onset of the random motion is established. The particles which execute random motions will be heated and their energy spread will increase. The width of the energy spectrum of the random motions is proportional to $\ln I_{b}$.

\section{REFERENCES}

[1] J. Gao, "Bunch lengthening and energy spread increasing in electron storage rings", Nucl. Instr. and Methods, A418 (1998), p. 332.

[2] J. Le Duff, CERN 85-19, p. 125.

[3] R.Z. Sagdeev, D.A. Usikov, and G.M. Zaslavsky,"Nonlinear physics from the pendulum to turbulences and chaos", Contemporary conceptes in physics, harwood academic publishers, 1988.

[4] B. Chirikov, Phys. Reports, Vol. 52, No. 5 (1979), p. 263379. 